\title{
Effects of Parameters of Photonic Crystal Fibre on Dispersion and Confinement
}

\author{
Shahid Masood Ahmad Khan ${ }^{1}$, Mohammad Shahid ${ }^{2}$
}

\author{
${ }^{1}$ Research Scholar, Department of ECE, Sri SatyaSai University of Technology \&Medical Sciences, Sehore, MP, India \\ ${ }^{2}$ Lecturer, Department of Information system, Faculty of Informatics, University of Gondar, Ethiopia
}

\begin{abstract}
A new design of hexagonal-lattice photonic crystal fiber, with solid core is proposed for study of dispersion and confinement loss for optical communication. The fiber has five air-hole rings, yet the diameter of air holes is different in their structures. In this work we observed the effect of varying $d 1$ (diameter of inner two rings) keeping $d 2$ constant, effect of varying $d 2$ (diameter of outer three rings) keeping $d 1$ constant and the variation of lattice constant $\Lambda$ on dispersion and confinement loss, and it is found that the effect of varying $d 1$ has a greater effect on dispersion in comparison with confinement loss and altering d2 has no significant effect on dispersion but have greater effect on confinement loss. Finite-difference time domain (FDTD) method is used for simulation.
\end{abstract}

Keywords-Photonic crystal fiber (PCF), Total internal reflection (TIR), Chromatic dispersion, Confinement loss.

\section{INTRODUCTION}

Development of wavelength division multiplexing (WDM) in recent years and the requirement of transmitting data over optical fibers at high bit rates and third wavelength window have caused serious concerns using conventional single mode fibers in optical communication. High dispersion in conventional single mode fibers leads to partial loss of data in long distances of data communication [1-4], thus researchers are interested to design a new optical transmission media with lower and uniform dispersion and low losses. The photonic crystal fiber is new class of fiber which are also referred as air-silica, holey or microstructured fiber.Their structures resemble those of the conventional fibers, as they comprise a core and a cladding, yet the cladding is of two dimensional photonic crystal types consisting of air hole arrays that run along the fiber length show unique properties, which are attractive for applications that cannot be realised by conventional fibers[5-6]. PCFs or holey fibers guide the light via one of two mechanisms: effective-index guidance and photonicband gap (PBG) guidance. In the PCFs with effective-index mechanism, and as the light is guided based on the total internal reflection between a solid core and a cladding region with multiple air-holes i.e. the light is confined in a higher refractive index region of core, instead, when the light is confined in a region with a refractive index lower than surrounding area of cladding, it is necessary the presence of the photonic band gap i.e., PCFs based on PBG have the capability to control the guidance of light within a certain frequency band as in hollow-core fibers[7]. One of the most important advantages offered by Photonic crystal fibers(PCFs) is the high design flexibility. In fact, by changing the geometric characteristics of the fiber crosssection, such as the air-hole dimension or hole to hole spacing [8].It is possible to design fibers with diametrically opposed optical properties. PCFs with unusual guiding, dispersion, and nonlinear properties can be designed and successfully used in various applications. The main drawback which affects this new kind of fibers is related to the attenuation, which is higher than that of conventional optical fibers [4]. Manufacturing process of Photonic crystal fibers is made by stacking tubes and rods of silica glass into a large structure (preform) of the pattern of holes required in the final fiber. The preform is then bound with tantalum wire and then is taken to a furnace of fiber drawing tower. The furnace is filled with argon and reaches temperature about $2000{ }^{\circ} \mathrm{C}$ as aftereffect the glass rods and tubes get soften. Later, the preform is fused together and reduced to $1 \mathrm{mmsize}$ with hole around $0.05 \mathrm{~mm}$ diameter. In other words by increasing the furnace temperature the air hole size can be reduced. Defects are created by replacing tubes for solid rods (as in the case of highly nonlinear PCF or by removing a group of tubes from the preform (hollow core photonic PCF). Since the fabrication process is quite potent, complicated geometries can be achieved. For instance the geometry of the centre defect can be modified by the introduction of the thicker and thinner tubes at different position around the defect[5]

\section{THEORETICAL DISCUSSION}

Effective mode index of a guided mode for a given wavelength is obtained by solving an Eigen value problem drawn from Maxwell's equations using the FDTD. The effective mode index, $\mathrm{n}_{\mathrm{eff}}$, can be obtained as [7-10]

$$
\mathrm{n}_{\mathrm{eff}}=\beta / \mathrm{k}_{0}
$$

Here, $\beta$ is the propagation constant and $k_{o}$ is the free space wave number. 
Effective mode index has both real and imaginary parts. The total dispersion is sum of the material dispersion and the waveguide dispersion. The chromatic dispersion, $D(\lambda)$, of a PCF is easily calculated from the second derivative of the real part of the effective mode index

$$
\mathrm{D}=-\frac{\lambda}{\mathrm{c}} \frac{\partial^{2} \mathrm{Re}\left(\mathrm{n}_{\mathrm{eff}}\right)}{\partial \lambda^{2}}
$$

Where $\operatorname{Re}($ neff) is the real part of neff, $\lambda$ is the wavelength in units of $\mu \mathrm{m}$, and $\mathrm{c}$ is the velocity of light in vacuum[810].

The material dispersion given by Sell meier's formula is directly included in the calculation. Therefore $D(\lambda)$ corresponds to the total dispersion of the PCF. The confinement losses, Lc, are because of the finite number of air-holes which can be made in the fibre cross-section. The confinement loss can be given by the imaginary part of the mode index as

$$
\mathrm{Lc}=8.686 \mathrm{k}_{0} \operatorname{Im}\left(\mathrm{n}_{\mathrm{eff}}\right)
$$

whereIm $\left(\mathrm{n}_{\text {eff }}\right)$ is the imaginary part of the effective mode index.

\section{DESIGN PARAMETER AND SIMULATION RESULT}

The primary design for proposed PCF is shown in figure-1. This PCF have five air hole rings with solid core. The diameter of the air holes in the inner two rings is considered to be $\mathrm{d} 1=0.65 \mu \mathrm{m}$ and diameter of air holes in three outer rings is equal to $\mathrm{d} 2=1.4 \mu \mathrm{m}$. The lattice structure of the cladding in this PCF is hexagonal or triangular and the spacing between the centres of adjacent holes (pitch), $\Lambda$, is $2.3 \mu \mathrm{m}$. For our study to see the behaviour of dispersion and confinement loss onaltering the dimension of air hole diameter and hole to hole spacing. Here we considered the value of $\mathrm{d} 1$ (diameter of inner two rings) to be $0.68 \mu \mathrm{m}$, $0.72 \mu \mathrm{m}$ and $0.76 \mu \mathrm{m}$, diameter of outer three rings $\mathrm{d} 2$ is constant and equal to $1.4 \mu \mathrm{m}$ and pitch is considered to be $2.3 \mu \mathrm{m}$.

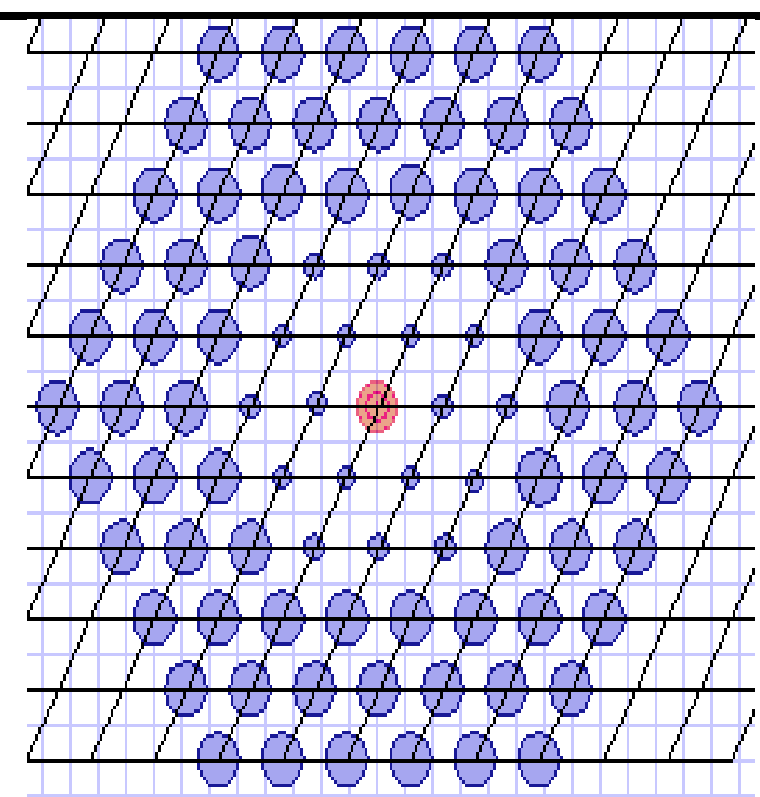

Fig.1: Photonic crystal fiber with triangular lattice structure

Figure-2shows the dependence of model effective index $n_{e f f}$ with respect to the wavelength $\lambda$, by fixing pitch $=2.3 \mu \mathrm{m}$ and changing $\lambda$ in the range of $0.7 \mu \mathrm{m}-2.0 \mu \mathrm{m}$ for these three variation of $\mathrm{d} 1$. It is found that that effective index decreases with the increase in wavelength and decreases with the increase in the $\mathrm{d} 1$ (inner two rings).

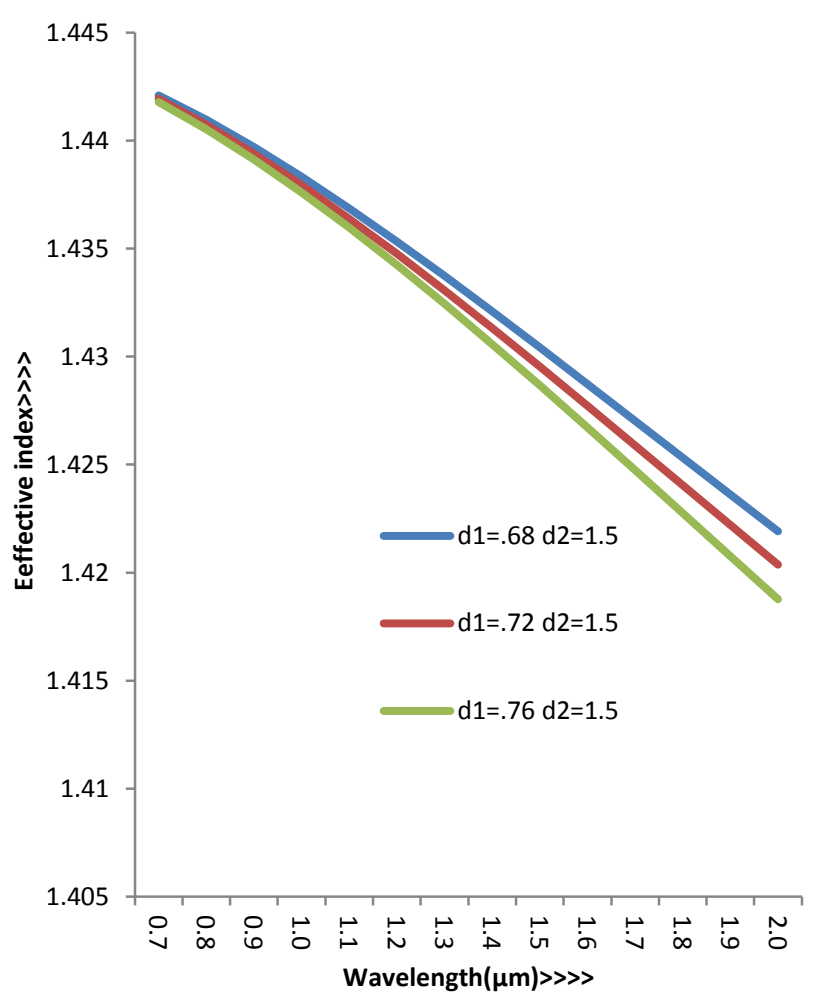

Fig.2: Effective index dependence with wavelength Figure-3 and Figure-4 show the dependence of chromatic dispersion and confinement loss on wavelength, which 
showthat value of chromatic dispersion increase with the increase in diameter d1.Among these three design it is found that minimum dispersion is $25.44234 \mathrm{ps} / \mathrm{nm}-\mathrm{km}$ with $\mathrm{d} 1=0.68 \mu \mathrm{mat} 1.5 \mu \mathrm{m}$ (mostly use optical wavelength).It shows thaton Increasing of $\mathrm{d} 1$ leads to smaller core area that may increase the confinement loss. On the other hand, as d1 increases air filling fraction also increases. Greater air filling fraction results in lower confinement loss. Therefore, d1 has no significant effect on confinement loss.

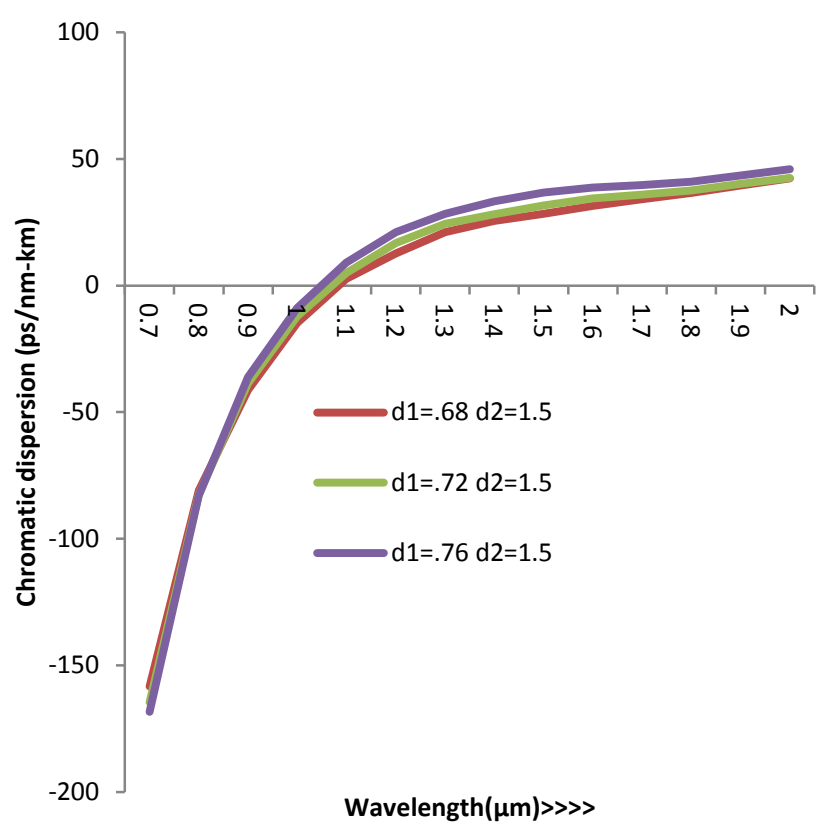

Fig.3: Chromatic dispersion of a triangular lattice PCF with varying diameter of inner two rings of air holes at $d 2=1.5 \mu \mathrm{m}$ and $\Lambda=2.3 \mu \mathrm{m}$

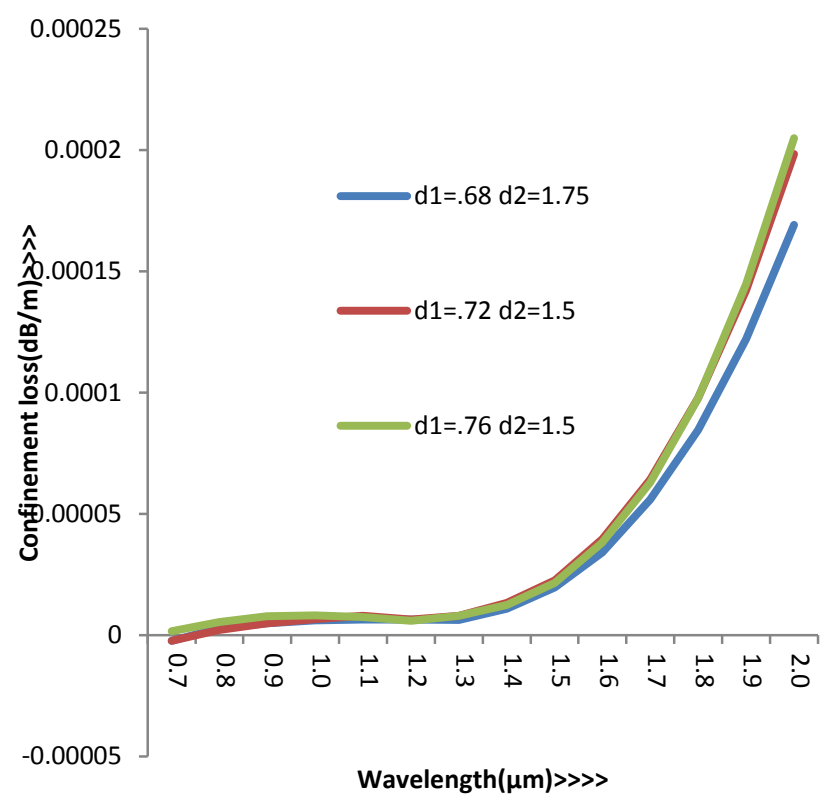

Fig.5: Confinement loss of a triangular lattice PCF with varying diameter of inner two rings of air holes at $d 2=1.5 \mu \mathrm{m}$ and $\Lambda=2.3 \mu \mathrm{m}$
Table.1: Effect of varying d1 on chromatic dispersion and confinement loss, $d 2=1.5 \mu \mathrm{m}$ and $\Lambda=2.3 \mu \mathrm{m}$

\begin{tabular}{|l|l|l|}
\hline $\mathrm{d} 1(\mu \mathrm{m})$ & $\begin{array}{c}\text { Chromatic dispersion } \\
\mathrm{ps} / \mathrm{nm}-\mathrm{km}\end{array}$ & $\begin{array}{c}\text { Confinement loss } \\
\mathrm{dB} / \mathrm{km}\end{array}$ \\
\hline 0.68 & 25.44234 & 0.0109 \\
\hline 0.72 & 28.25456 & 0.0113 \\
\hline 0.76 & 33.34336 & 0.0115 \\
\hline
\end{tabular}

Table1 above shows the effect of varying d1 (inner two rings) on chromatic dispersion and confinement loss $\mathrm{d} 2=1.5 \mu \mathrm{m}$ at $1.5 \mu \mathrm{m}$ wavelength, it is found that dispersion increases with the increase in $\mathrm{d} 1$ but there is minor variation in the confinement loss with the variation in diameter of inner rings.

\section{CONCLUSION}

So we have designed a new PCF and have studied the effect of altering the diameters of the air holes and adjacent hole spacing or pitch on dispersion and confinement loss characteristics in wavelength range from $0.7 \mu \mathrm{m}$ to $2.0 \mu \mathrm{m}$. From this we observed that on varying d1 (diameter of inner two rings) confinement loss hardly change but the dispersion and its slope change drastically, for higher value of $\mathrm{d} 1$ the dispersion curve shifts downward (below zero dispersion), changing the value of $\mathrm{d} 2$ (diameter of outer three rings ) has no more effect on dispersion but there is effect on confinement loss.Larger the value of air hole diameter lower the confinement loss. In last case we studied about the variation in pitch, which shows that dispersion slope is almost constant,but significantly affect the value of dispersion and confinement loss, when diameter of air holes constant, increasing $\Lambda$, result lower $\mathrm{d} / \Lambda$ ratio and refractive index of cladding rises hence confinement loss increases and shifts dispersion curve toward higher value.

\section{REFERENCES}

[1] J. C. Knight and P. S. J. Russell, "Photonic crystal fibers: New way toguide light," Science, vol. 296, pp. 276-277, 2002.

[2] J. C. Knight, T. A. Birks, P. S. J. Russell, and D. M. Atkin, "All-silicasingle-mode optical fiber with photonic crystal cladding," Opt. Lett.,vol. 21, pp. 1547-1549, 1996.

[3] T. A. Birks, J. C. Knight, and P. S. J. Russell, "Endlessly single-modephotonic crystal fiber," Opt. Lett., vol. 22, pp. 961-963, 1997.

[4] J. C. Knight, J. Broeng, T. A. Birks, and P. S. J. Russell, "Photonicband gap guidance in optical fibers," Science, vol. 282, pp. 1476-1478,1998. 
[5] T. Matsui, J. Zhou, K. Nakajima, and I. Sankawa, "Dispersion-flattenedphotonic crystal fiber with large effective area and low confinementloss," J. Lightw. Technol., vol. 23, no. 12, pp. 4178-4183, Dec. 2005.

[6] J.C.Knight, T.A.Birks, P.St.J.Russell, and J.P.de Sandro,"Properties of photonic crystal fiber and the effective indexmodel", J.Opt.Soc.Am., Vo1.15, No.3, 1998, pp.748-752.

[7] J.W.Fleming, "Material dispersion in lightguide glasses"Electr.Lett., Vo1.14, 1978, pp.326 328.

[8] Saitoh, K., M. Koshiba, T. Hasegawa, and E. Sasaoka, Chromatic dispersion control in photonic crystal- fibers: Application to ultra-fattened dispersion," Opt. Express, Vol. 11, 843-852, 2003.

[9] Saitoh, K. and M. Koshiba,Numerical modeling of photoniccrystal fibers," IEEE J. Lightwave Technol., Vol. 23, 3580\{3590,2005.

[10] Ligsgaard, J., A. Bjarklev, and S. E. B. Libori, Chromatic dispersion in photonic crystal fibers fast and accurate scheme forcalculation," J. Opt. Soc. Am. B, Vol. 20, 443-448, 2003. 